\title{
Impresión de diseños simétricos en la obra de Escher
}

\author{
Hugh Hilden* \\ José Montesinos** \\ Débora Tejada*** \\ Margarita Toro $* * * *$
}

Artículo recibido: 15-01-2012 y aprobado: 15-11-2012

\section{Stamping the symmetric designs of Escher}

Resumen: La búsqueda, por parte del ser humano, de bellos diseños, la selección de formas y colores de distintas piezas para sus muros y embaldosados, y la repetición sistemática de motivos produjeron patrones simétricos como ejemplos de teselados. Así mismo, la naturaleza ha encontrado bellísimos teselados resolviendo sus propios problemas. Un teselado embaldosado de una superficie es cubrirla con una misma pieza que se repite sin dejar espacios ni solapamientos. Aunque a simple vista se piense que son infinitas las formas de producir diseños simétricos planos, básicamente existen solo 17 formas de producirlos. Mostraremos que la ejecución de estos teselados sigue unas reglas sencillas y precisas, las cuales hemos utilizado para imaginar 17artefactos, los cuales son ejemplos del concepto debido a William Thurston, deorbifold (orbificie o calidoscopio generalizado) y que pueden ser utilizados en la impresión de cualquier diseño simétrico plano. Exhibiremos estos artefactos por medio de algunos dibujos y utilizaremos algunas de las obras de Escher para ilustrar nuestra conferencia. Se verá que los conceptos de translación, rotación y de reflexión pueden enseñarse fácilmente por medio de la utilización de estos artefactos.

Palabras clave: Grupos cristalográficos, simetría, teselados, orbifices, calidoscopios y Escher.
Abstract: In his need to find beautiful designs, forms and colors in the decoration of walls and the tiling of floors, humankind produced symmetric patterns that are examples of the concept of tessellation. In its own way, in order to solve its problems, nature had found wonderful tessellations. A tessellation or a surface tilling is the process of covering completely a surface with one type of tile that is repeated over and over without gaps or overlaps. Although it seems that there are infinitely many ways to produce symmetric plane designs, there are basically only 17 possible ways to produce a design. We will show that the execution of these tessellations follows some simple and concise rules, that we have used to construct 17 artifacts that can be used in the impression of any symmetric plane design. These artifacts are practical examples of Bill Thurston's concept of Orbifold. As an illustration of our presentation, we will exhibit these artifacts by using some pictures and some of Escher's designs. We will show that it is possible to teach, in an easy way, the concepts of rotation, translation and reflection by using these artifacts.

Keywords: Crystallographic groups, symmetry, tessellation, orbifold and Escher.

\footnotetext{
*Universidad de Hawaii: mike@math.hawaii.edu.

**Universidad Complutense de Madrid: jose_montesinos@mat.ucm.es.

***Universidad Nacional de Colombia: dtejada@unal.edu.co.

****Universidad Nacional de Colombia: 4mmtoro@unal.edu.co ${ }^{1}$ Una versión preliminar de este artículo ha sido publicada en las memorias del XX Encuentro de Geometría y sus
Aplicaciones, Universidad Pedagógica Nacional, Bogotá, 23 a 25 de junio de 2011 .
} 


\section{Introducción}

El estudio de sólidos cristalinos y del principio que gobierna su crecimiento, su forma externa y su estructura interna es llamado cristalografía. El orden interno o cristalino de un mineral se puede pensar como un motivo repetido sobre un retículo, que es un arreglo periódico de puntos en el espacio. Por esta razón en cristalografía es fundamental estudiar los patrones de estas simetrías en el espacio. Con el fin de ayudar a entender estos patrones en tres dimensiones, es importante estudiar el caso plano, que es el objetivo principal de este trabajo.

Introducimos el tema dando un recorrido muy rápido en la vida de Escher. Luego nos centraremos en los aspectos matemáticos de los teselados. Un teselado o embaldosado de una superficie consiste en cubrirla con una misma pieza que se repite sin dejar espacios ni solapamientos. Aunque a simple vista se piense que son infinitas las formas de producir diseños simétricos planos, básicamente existen solo 17 formas de producirlos, cada una de ellas asociada a un grupo cristalográfico plano. Mostraremos que la ejecución de estos teselados sigue unas reglas sencillas y precisas, las cuales permiten imaginar 17 artefactos que están en correspondencia biunívoca con los 17 grupos cristalográficos planos y que pueden ser utilizados en la impresión de cualquier diseño simétrico plano. Veremos que los conceptos de translación, rotación y de reflexión pueden enseñarse fácilmente por medio de la utilización de estos artefactos. Por otro lado, cada uno de estos artefactos es la ejemplificación concreta de un orbifold euclídeo y, por tanto, ellos nos permiten acceder en una forma práctica al concepto de orbifold.

$\mathrm{Al}$ lector, le recomendamos no alejarse del computador, pues la lectura se debe hacer interactuando con muchos sitios web'.

\section{Notas biográficas sobre Escher}

Los dibujos de Escher muestran una variada gama de paradojas y objetos imposibles, y exploran los alcances de las ilusiones ópticas. Esta obra, reconocida por su realismo meticuloso y admirada por su virtuosismo, no cesa de intrigar a matemáticos, psicólogos y al público general.

Comenzaremos recordando muy rápidamente quién fue Maurits Cornelis Escher. Nació en Leeuwarden (Frise, Países Bajos) en 1898. Entre 1903 y 1918 realizó sus estudios de primaria y secundaria. Con respecto a estos años hay que decir que la secundaria fue prácticamente una pesadilla para él, con la única excepción de las dos horas semanales de dibujo que disfrutaba enormemente. En 1919 entró a la Escuela de Arquitectura y Bellas Artes de Haarlem, donde rápidamente se dio cuenta que su inclinación no era la Arquitectura sino las Bellas Artes.

Su maestro, el profesor De Mesquita, fue su guía en el aprendizaje del difícil arte del grabado. Para 1922 ya había adquirido gran destreza en el manejo de técnicas de grabado. Su maestro consideró que ya estaba maduro y que era hora de que emprendiera su camino

\footnotetext{
Para apreciar algunos videos sobre los artefactos que imprimen diseños simétricos, recomendamos una visita a la página web: http://www.atractor.pt
} 
solo. Escher seguiría en contacto con De Mesquita hasta su muerte.

En el periodo comprendido entre 1922 y 1935 vivió en Italia, donde conoció a Jetta Umiker, quien sería su esposa desde 1924 y con quien compartió la pasión por dibujar y pintar. Cada primavera emprendía largas excursiones por los pueblos de Italia. La obra de estos años se enmarcó principalmente en el tema de paisajes y grabados de pequeños pueblos, inspirados en las múltiples excursiones hechas en Italia. Para esa época, Escher era aún muy desconocido.

En 1935 el clima fascista que se vivía en Italia le hace la vida insoportable y decide dejar Italia. Siendo Jetta de origen Suizo, se radican en Suiza por corto tiempo y en el año siguiente hacen varios cruceros por el Mediterráneo. Estos los financiaron con grabados de Escher, ya que el dueño de la compañía de cruceros, quien era un amante del arte, le aceptó el canje que Escher le propuso en una carta. En uno de estos cruceros visitó por segunda vez el Palacio de la Alhambra de Granada. Ya había estado allí diez años antes. Esta segunda visita le entregó a Escher las llaves de un mundo lleno de nuevas posibilidades. En compañía de su esposa tomó nota cuidadosa de muchos de los diseños de la Alhambra, notas que serían su inspiración en los años venideros.

En Internet se pueden observar los primeros dibujos de teselados inspirados en la Alhambra. Allí vemos, por ejemplo, las acuarelas conocidas como Niño chino (China boy), Ardillas (Squirrels) y Hombres fuertes (Strongmen). Todas ellas son de1936.

En 1937 se instaló en Uccle, cerca de Bruselas y en sus obras se ve ya la influencia de la Alhambra. En la misma página web podemos apreciar cómo él comienza a hacer variaciones en los teselados para producir obras más complejas. Por ejemplo, en 1937 produce los grabados Desarrollo I (Development I) y Metamorfosis I (Metamorphosis I) en donde se ve claramente la utilización dinámica que él hace de los teselados.

En enero de 1941 se trasladó para Baarn en los Países Bajos. Su clima frío y húmedo obligó a Escher a trabajar en recogimiento, lo que a su vez dio pie para que allí fuese la cuna de su obra más rica. En 1962 debió ser operado y su producción se suspendió por un corto periodo. En 1969 terminó su última y magistral xilografía: Serpientes (Serpents). Esta xilografía está llena de detalles; se pueden apreciar algunos de menos de $2 \mathrm{~mm}$. Murió el 21 de febrero de 1971. Un estudio muy completo de la vida y obra de M. C. Escher se puede leer en Le miroir magique de M.C. Escher (Ernst, 1978).

\section{Escher: ¿matemático o artista?}

Cuando se observan las obras de Escher, uno no puede dejar de maravillarse por sus minuciosos detalles. Su obra muestra su carácter obsesivo e incansable. En el momento en que Escher se dio cuenta y reconoció la riqueza escondida en los diseños geométricos de la Alambra, comenzó un trabajo permanente y repetitivo sobre ellos. Hizo investigaciones matemáticas que lo llevaron a comprender y a descubrir muchos trucos que él pudo aplicar. Los matemáticos, como el geómetra Coxeter, se fascinaron con la obra de Escher e incluso lo invitaron a hablar en congresos de matemáticos, donde Escher no podía más que celebrarlo dado que se consideraba negado para 
las matemáticas. Podemos afirmar, tal como él mismo lo hacía, que no era matemático, pues no manejaba el lenguaje formal que usamos los matemáticos; sin embargo, sin temor a equivocarnos, podemos decir con toda seguridad que la mente de Escher era privilegiada en su concepción del mundo desde un punto de vista geométrico. Era simplemente un artista dotado con una profundamente geométrica.

La obra de Escher se puede dividir en tres temas diferentes:

- Paisajes.

- Teselados o embaldosados.

- Mundos imposibles (juegos con la perspectiva). El libro Un mundo de figuras imposibles (Ernst, 1991) es un muy buen compendio de estos trabajos de Escher.

\section{Teselados o embaldosados}

Solo los dos últimos temas tienen interés para nosotros desde el punto de vista matemático. El tema de los mundos imposibles merece un estudio completo pero este no es de nuestro interés en este espacio. Aquí queremos estudiar los teselados y tratar de entender las herramientas matemáticas que se usan en el aparentemente complejo y misterioso mundo de ellas.

Un teselado embaldosado de una superficie consiste encubrirla con una misma pieza (baldosa) que se repite sin dejar espacios ni solapamientos. Mostraremos, sin demeritarla complejidad del arte de Escher, que la ejecución de estos teselados sigue unas reglas sencillas y precisas.

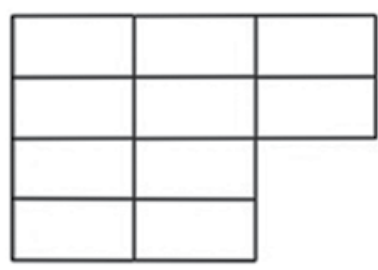

Figura1. Teselado con rectángulos

A lo largo de la historia de la civilización se encuentran muchos ejemplos de teselados o mosaicos. Desde que el hombre comenzó a construir paredes, pisos y techos, la colocación de piedras, ladrillos o baldosas produjeron en forma natural el surgimiento de teselados. La búsqueda de bellos diseños, la selección de formas y colores de las piezas y la repetición sistemática de motivos produjeron patrones simétricos como ejemplos de teselados.

Aunque se tienen infinitas formas de hacer diseños simétricos, básicamente existe un número muy restringido de patrones. Hay solo 17 patrones básicos para hacer diseños simétricos sobre un plano. En efecto, el cristalógrafo E. S. Fedorov, interesado en el estudio de las formas de los cristales, probó en 1891 que existen solamente 17 grupos cristalográficos planos. Para probar esto es suficiente considerar solo cuatro tipos de movimientos rígidos de una baldosa sobre un plano (decimos que son rígidos porque no cambian el tamaño de la baldosa ni la deforman, simplemente la cambian de lugar sobre el plano). Ellos son: traslaciones, rotaciones, reflexiones y reflexiones corridas. Cualquier movimiento rígido que nos imaginemos puede ser reproducido con una combinación apropiada de estos movimientos. En realidad, sería suficiente considerar solo el movimiento de reflexión, ya que una traslación se puede producir a partir 
de la combinación de dos reflexiones a lo largo de dos ejes paralelos, una rotación también es la combinación de dos reflexiones, pero en este caso los ejes no son paralelos y una reflexión corrida es simplemente el producto de una reflexión por una traslación. Estas cuatro clases de movimientos generan grupos algebraicos. Estos grupos son solamente 17 (salvo homomorfismos) y son llamados los grupos cristalográficos planos o euclídeos.

El entendimiento en dos dimensiones permitió que un poco más adelante Fedorov, Schonflies y Barlow (estos dos últimos matemáticos) demostraran la existencia de solamente 230 grupos cristalográficos tridimensionales.

Las aplicaciones de los grupos cristalográficos han sido diversas: el arte, la arquitectura y hasta la antropología se han beneficiado de ellos. Por ejemplo, como aplicación en la antropología, Albis-González y Valencia-Oviedo (1990) usan los grupos de simetría planos para confirmar la existencia de divisiones estilísticas en la cerámica precolombina de la región central de Panamá.

En la figura 1 vemos un teselado euclídeo con rectángulos. Observándola es natural preguntarse: ¿será posible teselar un plano con baldosas que no sean polígonos? La respuesta es sí, y Escher fue un maestro en la producción de teselados a partir de baldosas en forma de figuras humanas o de los más diversos y extraños animales. El arte nazarí, que podemos apreciar en los palacios, como en la Alhambra de Granada, fue rico en la creación de teselados, pero por razones de índole religiosa, ellos no se permitieron el uso de figuras humanas o de animales (véase Montesinos-Amilibia, 2005).

\section{Calidoscopios}

Hemos dicho que queremos enseñar cuáles son las reglas simples que hay veladas detrás de los 17 patrones de diseños de simetría sobre un plano. Comenzaremos con un ejemplo que todos conocemos: un calidoscopio. Este es un artefacto formado por espejos que tiene la propiedad que con el mismo artefacto se pueden producir infinitos diseños simétricos sobre el plano, simplemente cambiando la imagen que se mira a través de él. Para que el calidoscopio funcione y no se solapen las figuras unas sobre otras, los espejos deben formar entre sí ángulos especiales. Por ejemplo, si los espejos forman un triángulo equilátero, la obra de Escher llamada Lagarto, pez y murciélago (Lizard/Fish/Bat) se puede reproducir mirando una baldosa triangular que contiene únicamente las mitades del lagarto, del pez y del murciélago. En la figura 2 representamos un esquema de dicha baldosa con su diseño simétrico.

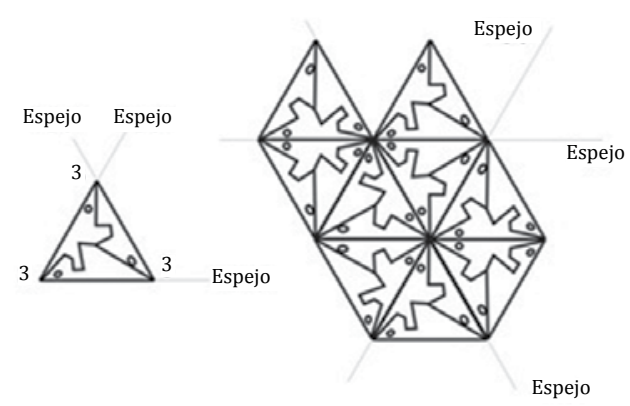

Figura 2. Baldosa y diseño de calidoscopio de triángulo equilátero

El diseño producido por un calidoscopio triangular con tres ángulos de $60^{\circ}$ corresponde al grupo cristalográfico euclídeo denotado por p3m1, que es generado como grupo algebraico por 
tres reflexiones a lo largo de tres ejes que forman un triángulo equilátero. En este mismo diseño se ilustran (figura 3) los movimientos rígidos de traslación, rotación, reflexión y de reflexión corrida, de una baldosa básica.
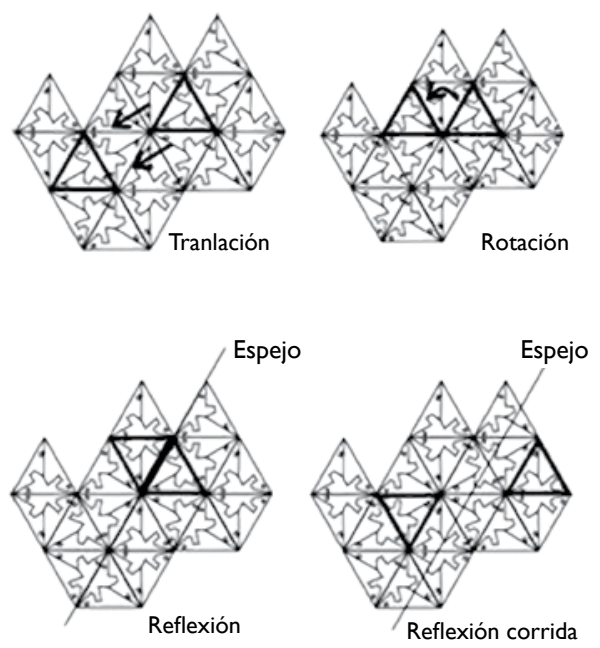

Figura 3. Movimientos rígidos aplicados a una baldosa

Para estudiar diseños simétricos hay dos puntos de vista posibles. De un lado, podemos usar el lenguaje algebraico, y en este sentido es que hablamos de grupos cristalográficos o, por otro lado, nos es permitido usar el lenguaje geométrico y topológico, y en este caso nos referimos en términos de orbificies, "orbifolds" o calidoscopios generalizados. En este artículo no definimos explícitamente el concepto de orbifold, pues no es nuestro objetivo. Nos limitaremos a dar los 17 orbifolds asociados a los grupos cristalográficos planos. Por simplicidad en el lenguaje, el término que usaremos será el de calidoscopio; entendiendo que nos referiremos a los calidoscopios generalizados y que los calidoscopios formados por espejos son solo ejemplos particulares.

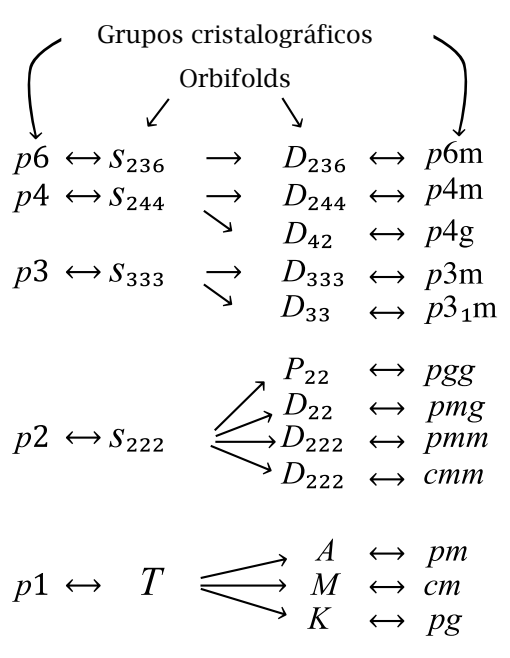

Figura 4. Grupos cristalográficos vs.orbifolds

Históricamente, la simetría de las teselaciones fue primero entendida desde el punto de vista algebraico. Como ya lo hemos dicho, los trabajos del cristalógrafo E. S. Fedorov probaron en 1891 la existencia de solo 17 grupos algebraicos (cristalográficos) que permiten entender los diseños simétricos sobre un plano. A partir de las ideas de W. Thurston (1997), la simetría de los diseños simétricos se entiende también utilizando el concepto de calidoscopio, que pertenece al mundo de la geometría y de la topología. Ambos lenguajes son equivalentes; para cada grupo cristalográfico existe un calidoscopio y viceversa. En la figura 4 listamos los 17 grupos cristalográficos euclídeos con sus correspondientes calidoscopios. Usamos las notaciones usuales para los grupos cristalográficos y para los calidoscopios u orbifoldseuclídeos. La notación de los orbifolds se irá entendiendo a medida que se estudia cada uno de ellos (véase Klein y Hurlbut, 1999). 


\section{Movimientos rígidos y artefactos que los producen}

A continuación mostraremos cómo construir artefactos que permiten imprimir una misma imagen que se repite, obedeciendo al efecto de alguno de los movimientos rígidos (para mayores detalles véase Hilden, Montesinos, Tejada y Toro, 2011).

\section{Traslación}

Las rotativas utilizadas en la imprenta son un ejemplo del artefacto que produce traslaciones de una imagen. Cuando se tiene un rectángulo, hecho de material flexible, hay dos maneras de pegar las parejas de lados opuestos para formar dos cilindros diferentes. Y el efecto de impresión (figura 5) usando ambos cilindros es muy diferente. Uno producirá traslaciones horizontales mientras que el otro las producirá en forma vertical.
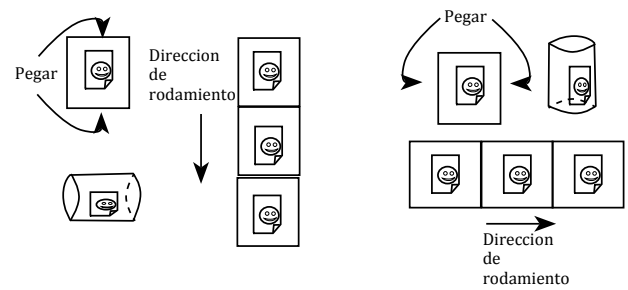

Figura 5. Traslaciones horizontales y verticales

\section{Rotación}

Para producir rotaciones necesitamos un artefacto en forma de cono, que al impregnarlo de tinta y ponerlo a rodar sobre un plano reproduzca el movimiento rígido de una rotación. Construimos el cono a partir de un sector circular con un ángulo de $360^{\circ} / \mathrm{n}$, donde $\mathrm{n}$ es un entero positivo, con el fin de que, al ir imprimiendo, no se solapen las imágenes. La figura 6 representa el caso de una rotación de $90^{\circ}$.

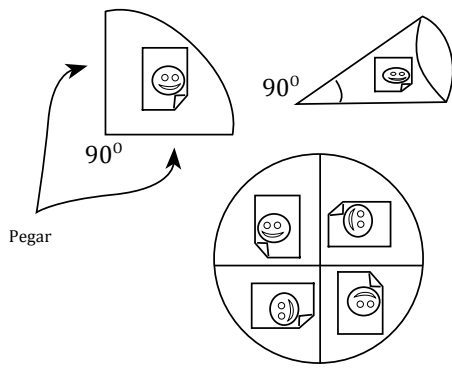

Figura 6. Rotación de $90^{\circ}$

\section{Reflexión}

En este caso, necesitaremos un artefacto que imite el efecto de un espejo (figura 7). Para esto podemos recurrir al arte de la serigrafía, que usa material poroso y delgado con el fin de poder imprimir una figura por cualquiera de los dos lados del material. Al hacer esto se obtiene la reflexión de la figura que se ve del otro lado, de igual forma, como si la estuviésemos mirando en un espejo. Este artefacto lo debemos hacer con un material delgado y poroso pero con cierta rigidez que permita el rodamiento. Además, el artefacto debe tener bordes rectos, que actuarán como ejes de reflexión y sobre los cuales nos apoyamos para darle vuelta al artefacto y poder ir imprimiendo por ambos lados. Cuando el artefacto va rodando siempre se gira sobre alguna arista manteniéndola apoyada sobre el plano.
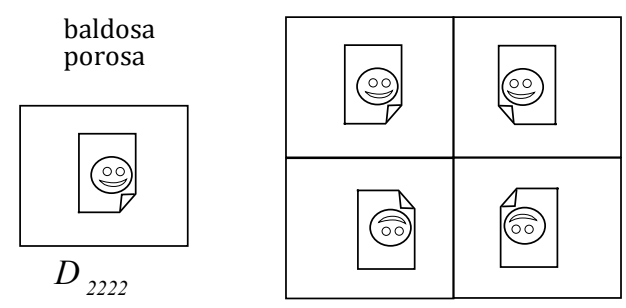

Figura 7. Reflexiones 


\section{Reflexión corrida}

Para reproducir una reflexión corrida necesitamos un artefacto que combine la posibilidad de hacer una reflexión, o sea que podamos imprimir por ambos lados y que, además, produzca también el efecto del cilindro en cuanto a traslaciones. Una banda de Möebius cumple ambas condiciones. Construimos una banda de Möebius a partir de un rectángulo hecho de material poroso y flexible, al cual se le pegan dos lados opuestos en forma cruzada. En la figura 8 mostramos la construcción de una banda y el efecto al imprimir con ella.
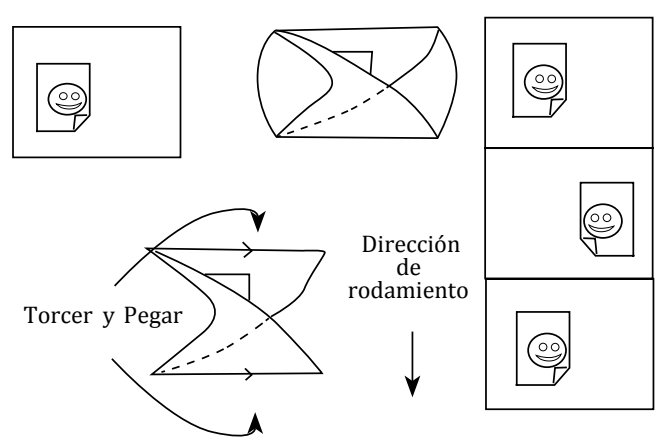

Figura 8. Cinta de Möebius y reflexión corrida

Así pues, cada movimiento rígido puede ser reproducido por un artefacto. Como cada grupo cristalográfico es generado por algunos de estos movimientos rígidos, una buena combinación de los artefactos anteriormente descritos nos dará la posibilidad de tener 17 artefactos diferentes, uno para cada uno de los 17 grupos cristalográficos. Estos artefactos son justamente los 17 calidoscopios generalizados u orbificies.

\section{Test para identificar el calidoscopio que corresponde a un diseño simétrico}

(Compárese el test de la tabla 1 con la figura 9 de Albis-González y ValenciaOviedo,1990.Véase también GutiérrezSantos, 1992 y Klein y Hurlbut, 1999).

\begin{tabular}{|c|c|c|}
\hline \multicolumn{3}{|r|}{ Caso I. No hay rotaciones: } \\
\hline I. & \multicolumn{2}{|r|}{ No hay reflexiones ni reflexiones corridas:Toro $\mathrm{T}$} \\
\hline 2. & \multicolumn{2}{|r|}{$\begin{array}{l}\text { No hay reflexiones pero hay reflexiones corridas: } \\
\text { Botella de Klein } \mathrm{K}\end{array}$} \\
\hline 3. & \multicolumn{2}{|c|}{$\begin{array}{l}\text { Hay reflexiones y reflexiones corridas: Cinta de } \\
\text { Möebius } M\end{array}$} \\
\hline 4. & \multicolumn{2}{|r|}{ Hay reflexiones y no reflexiones corridas:Anillo A } \\
\hline & \multicolumn{2}{|r|}{$\begin{array}{l}\text { Caso } 2 \text {. Hay rotaciones pero no hay reflexiones ni } \\
\text { reflexiones corridas }\end{array}$} \\
\hline I. & \multicolumn{2}{|c|}{ Hay centro de orden $6: S_{236}$} \\
\hline 2. & \multicolumn{2}{|r|}{ Hay centro de orden 3 pero no de orden $6: S_{333}$} \\
\hline 3. & \multicolumn{2}{|c|}{ Hay centro de orden $4: \mathrm{S}_{244}$} \\
\hline 4. & \multicolumn{2}{|c|}{ Solo hay centros de orden $2: \mathrm{S}_{2222}$} \\
\hline \multicolumn{3}{|c|}{ Caso 3. Hay rotaciones y reflexiones o reflexiones corridas } \\
\hline I. & \multicolumn{2}{|c|}{ Hay centro de orden $6: D_{\overline{236}}$} \\
\hline 2. & \multicolumn{2}{|r|}{$\begin{array}{l}\text { Solo hay centros de orden } 3 \text { y por todos ellos pasan } \\
\text { reflexiones: } D_{\overline{333}}\end{array}$} \\
\hline 3. & \multicolumn{2}{|r|}{$\begin{array}{l}\text { Solo hay centros de orden } 3 \text { y por alguno de ellos no } \\
\text { pasan reflexiones: } D_{3 \overline{3}}\end{array}$} \\
\hline 4. & \multicolumn{2}{|r|}{$\begin{array}{l}\text { Hay centros de orden } 4 \text { y por ellos pasan reflexiones: } \\
D_{\overline{244}}\end{array}$} \\
\hline 5. & \multicolumn{2}{|r|}{$\begin{array}{l}\text { Hay centros de orden } 4 \text { pero por ellos no pasan } \\
\text { reflexiones: } D_{4 \overline{2}}\end{array}$} \\
\hline 6. & \multicolumn{2}{|c|}{ Solo hay centros de orden dos } \\
\hline & $\mathrm{I}$. & Por todos ellos pasan reflexiones: $\mathrm{D}_{\overline{222}}$ \\
\hline & 2. & Solo por algunos pasan reflexiones: $D_{2 \overline{22}}$ \\
\hline & 3. & $\begin{array}{l}\text { Por ninguno de ellos pasan reflexiones pero hay } \\
\text { reflexiones: } D_{22}\end{array}$ \\
\hline & 4. & $\begin{array}{l}\text { Por ninguno de ellos pasan reflexiones pero hay } \\
\text { reflexiones corridas: } \mathrm{P}_{22}\end{array}$ \\
\hline
\end{tabular}

Tabla 1. Test para identificar el calidoscopio que corresponde a un diseño simétrico 


\section{Teselados planos}

En lo que sigue estudiaremos los grupos cristalográficos junto con sus calidoscopios asociados (según la figura 4). Además los relacionaremos con algunas de las obras de Escher.

\section{Los grupos cristalográficos cm y pm y los calidoscopios M y A}

Si miramos detenidamente la obra Escarabajos $^{2}$ (Scarabs), sin tener en cuenta sus colores, podemos distinguir un eje de reflexión que pasa por el medio de los escarabajos y otro eje de reflexión corrida que pasa en medio de las patas de los escarabajos. Como no hay rotaciones, deducimos entonces que se trata del calidoscopio denotado por M (primera letra de Möebius). Este lo llamamos cinta de Möebius, la cual es la misma descrita anteriormente pero, además, imprimimos con ella misma varias cintas paralelas así: una vez se ha impreso una cinta como la de la figura 8 , se consigue la impresión de su reflejo en sus bordes al darle la vuelta a la cinta de Möebius como si fuese un calcetín. El calidoscopio $\mathrm{M}$ corresponde al grupo cristalográfico $\mathrm{cm}$, que es generado por una reflexión y una reflexión corrida con ejes paralelos.

Si en la misma obra de los escarabajos, tenemos en cuenta los colores, podemos distinguir dos ejes de reflexión paralelos, uno que pasa por la mitad de los escarabajos blancos y otro que pasa por el medio de los escarabajos cafés. Tenemos entonces dos ejes de reflexión paralelos y como no hay rotaciones, deducimos que el calidoscopio corres-

2 Véase http://www.tessellations.org/eschergallery20.htm pondiente es A, llamado anillo. Un anillo es un cilindro hecho de material poroso. La manera de operar con este cilindro es la de imprimir primero una cinta como cualquiera de las de la figura 5 y a continuación darle la vuelta al cilindro como a un calcetín para imprimir el reflejo de la primera cinta en una cinta paralela.

\section{El grupo cristalográfico $p 1$ y el toro $T$}

En la obra Pegasos ${ }^{3}$ podemos ver que hay una baldosa básica en forma de pegaso (en nuestro análisis no hacemos caso a los colores y pensamos que todos los pegasos son del mismo color) que se repite trasladada en dirección horizontal y en dirección vertical. Es decir, tenemos traslaciones en dos sentidos. Obsérvese que se ven también traslaciones diagonales, pero estas aparecen como composición de traslaciones horizontales con verticales. Observamos también que no hay rotaciones ni reflexiones. A partir de la baldosa del pegaso y teniendo la autorización para hacer traslaciones en dos sentidos (independientes) podemos teselar todo el plano. El grupo cristalográfico generado por dos traslaciones es p1 y corresponde al calidoscopio llamado toro, denotado por T. En la figura 9 exhibimos un toro y el diseño que se imprimiría con él.

Abrimos un paréntesis en nuestra discusión sobre los artefactos que nos permiten reproducir los teselados para hacer caer en cuenta cómo Escher se las ingenió para hacer sus diseños sin tener que usar artefactos como el toro. Aunque Escher no conocía el toro, supo aplicar la esencia del toro, el cual tiene

3 Ver http://britton.disted.camosun.bc.ca/jbescher3. htm y http://www.tessellations.org/eschergallery25.htm 
la particularidad de trasladar una figura en dos direcciones.
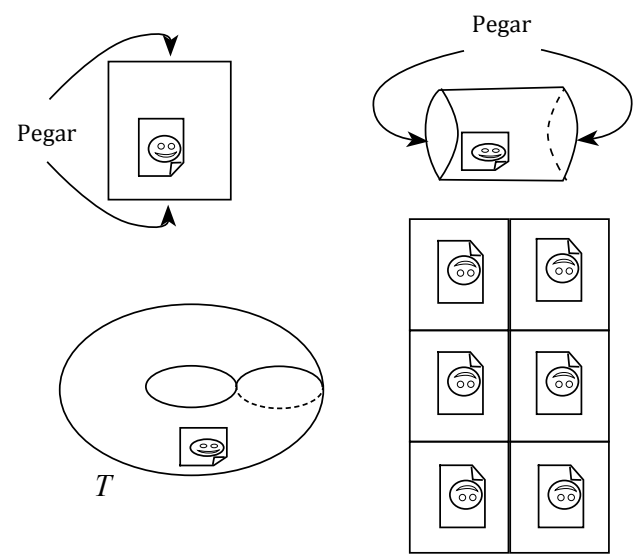

Figura 9. Diseño impreso con un toro

En la misma página web (http:// britton.disted.camosun.bc.ca/jbescher3. htm) se ve la animación que ilustra cómo se construye la baldosa básica a partir de un cuadrado y que probablemente nos muestra lo que Escher hizo. Allí podemos ver las dos traslaciones que hemos mencionado. La clave de esta construcción es la de partir de un teselado sencillo del plano hecho en este ejemplo por cuadrados y luego cada cuadrado se modifica tal cual se ve en la animación mencionada. La idea es deformar, por ejemplo, la línea que forma el lado superior del cuadrado, en este caso, se dibuja el perfil de la cabeza y del lomo del pegaso, para trasladarla enseguida hacia el lado inferior del cuadrado, formando ahora el pecho y las patas traseras del pegaso. A continuación se deforma la línea del lado derecho del cuadrado obteniendo las alas y la cola del pegaso para luego trasladarla hacia el lado izquierdo del cuadrado obteniendo con ella las patas delanteras. Trucos similares a este fueron utilizados por Escher para poder confeccionar sus diseños. En general, él partía de un teselado hecho por polígonos y a continuación los deformaba utilizando éste u otros trucos similares que más adelante comentaremos. El método anterior es posible aplicarlo siempre que se tengan dos lados paralelos e iguales en el polígono al cual se le hacen las deformaciones ${ }^{4}$.

Cerrando el paréntesis, en http:// www.tessellations.org/eschergallery15. htm encontramos la obra Sapos y peces de 1942 de Escher que podría ser impresa por un toro. En ella observamos que la baldosa básica está conformada por la pareja de un sapo junto con un pez y que, además, las traslaciones no son horizontales ni verticales, como en el caso de los pegasos, sino que siguen la dirección de dos diagonales, que no son perpendiculares entre sí. En este caso debemos construir el toro a partir de un paralelogramo que no es rectángulo. La dirección de sus lados nos dirá cuál es la dirección de las traslaciones. La figura 10 nos enseña un cilindro que genera traslaciones sesgadas.

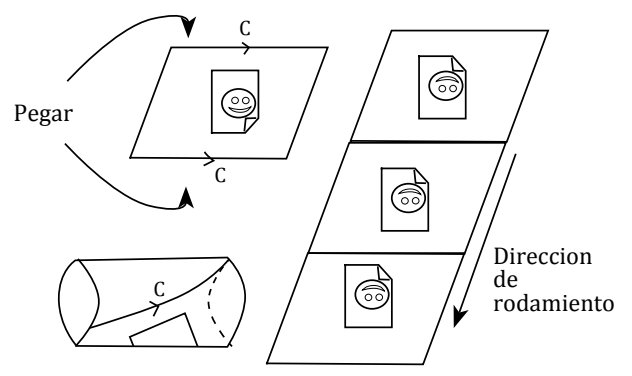

Figura 10. Traslación siguiendo una diagonal

Le recomendamos al lector que haga el ejercicio de construir este cilindro con un paralelogramo de papel.

\footnotetext{
4 Véase el mismo truco aplicado en la construcción de otras teselaciones (en particular las teselaciones 75 y 97) en: http://britton.disted.camosun.bc.ca/ jbescher4.htm, aunque en estos dos casos no se aplica sino a un solo par de lados.
} 
Los grupos cristalográficos p3m1, pmm, p4m y p6m y los cuatro calidoscopios comunes $D_{\overline{333}}, D_{\overline{2222}}, D_{\overline{244}}$ y $\overline{D_{\overline{236}}}$

El grupo cristalográfico p3m1 generado por reflexiones a lo largo de tres ejes que forman un triángulo equilátero y el grupo pmm generado por reflexiones a lo largo de cuatro ejes que forman un rectángulo están en correspondencia con los calidoscopios comunes formados por espejos y denotados por $D_{\overline{333}}$ y $D_{\overline{2222}}$, donde la letra $\mathrm{D}$ significa que se tiene un disco topológico con bordes y cada uno de los números indica que hay un ángulo en el borde de $180^{\circ} / \mathrm{n}$ formado por dos espejos consecutivos.

Ya discutimos anteriormente que la obra de Escher llamada Lagarto, pez y murciélago puede ser reproducida observando una baldosa triangular que contiene únicamente la mitad del lagarto, la mitad del pez y la mitad del murciélago a través de un calidoscopio de tres espejos que forman entre ellos ángulos de $60^{\circ} \mathrm{y}$, por tanto, este diseño corresponde al calidoscopio $\mathrm{D}_{\overline{333}}$ (figura 2). Por otro lado, la obra Motivos $4^{5}$ (Motifs 4) corresponde al calidoscopio $D_{\overline{2222}}$ y puede ser reproducida al mirar una baldosa cuadrada, que contenga solamente la mitad de una abeja, de una mariposa, de un pez y de un murciélago, a través de un calidoscopio formado por cuatro espejos que forman un cuadrado. Por otro lado, los calidoscopios $D_{\overline{244}}$ y $\mathrm{D}_{\overline{236}}$ corresponden a los grupos $\mathrm{p} 4 \mathrm{~m} \mathrm{y}$ p6m que son generados, cada uno por tres reflexiones a lo largo de tres ejes. En el primero los tres ejes forman un

5 Véase http://www.tessellations.org/eschergallery19.htm triángulo isósceles recto y en el segundo forman un triángulo con ángulos de $90^{\circ}$, $60^{\circ}$ y $30^{\circ}$. Al igual que los dos calidoscopios anteriores, sus diseños se pueden producir por tres espejos que formen los ángulos respectivos. En la figura 11 se ilustra $D_{\overline{2222}}$

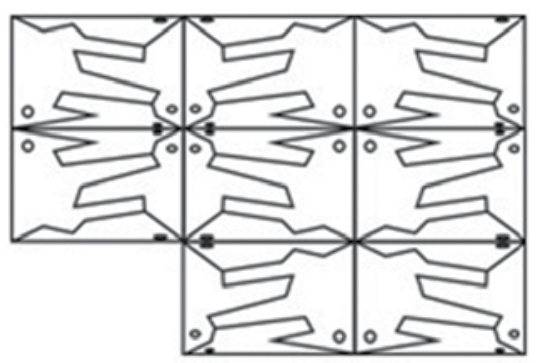

Figura 11. Diseño de calidoscopio $D_{\overline{2222}}$

\section{El grupo cristalográfico p3 y el calidoscopio $S_{333}$}

En la obra Reptiles ${ }^{6}$ vemos que hay una baldosa básica en forma de lagarto que se repite. En la misma forma que en el caso de los pegasos en nuestro análisis no nos interesa diferenciarlos colores. La baldosa se repite por rotación de $120^{\circ}$ en los vértices señalados con triángulos en la figura 12, y esto nos permite teselar todo el plano.

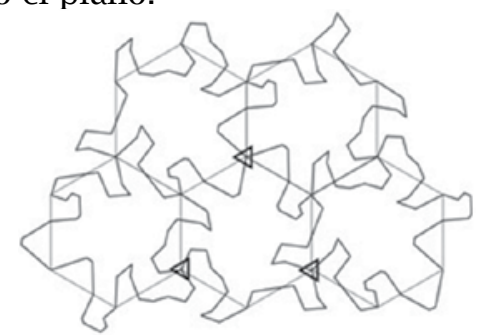

Figura 12. Centros de rotación de $120^{\circ}$

El grupo cristalográfico generado por tres rotaciones de $120^{\circ}$ en los vér-

\footnotetext{
6 Véase http://aixa.ugr.es/escher/800×600/Reptiles_(boceto).jpg
} 
tices de un triángulo equilátero es p3 y

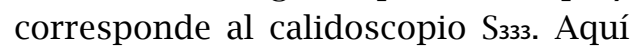
la letra $S$ nos indica que se tiene una esfera topológica y cada número $\mathrm{n}$ nos dice que hay un cono de $360^{\circ} / \mathrm{n}$. Recordemos que el artefacto que nos reproduce una rotación es un cono. Así, S3з3 es un artefacto parecido a una almohada o cojín triangular con tres esquinas, que a su vez son conos de $120^{\circ}$ cada uno. Lo mostramos en la figura 13.

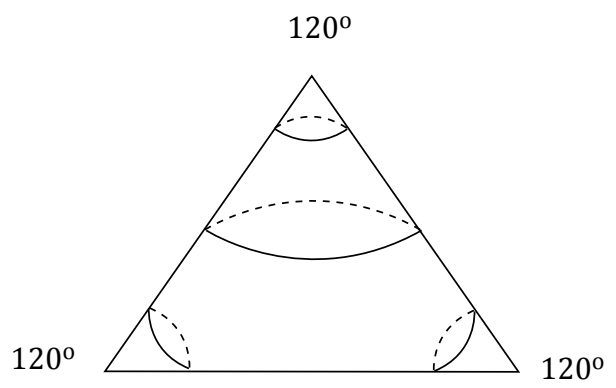

Figura 13. El cojín S333

Abramos de nuevo un paréntesis y expliquemos brevemente el truco que probablemente usó Escher para construir la baldosa del lagarto. Escher partió de una teselación hecha de hexágonos regulares y cada uno de estos hexágonos lo modificó teniendo en cuenta la esencia del calidoscopio $S_{333}$, que es la de producir tres rotaciones de $120^{\circ}$ en los vértices de un triángulo equilátero. La secuencia exhibida en la figura 14 nos ilustra los dos primeros pasos en la construcción de la baldosa básica a partir de un hexágono. No ilustramos el tercer paso que se realiza de manera similar para modificar los últimos dos lados del hexágono (Seymour y Britton, 1989, p. 207).
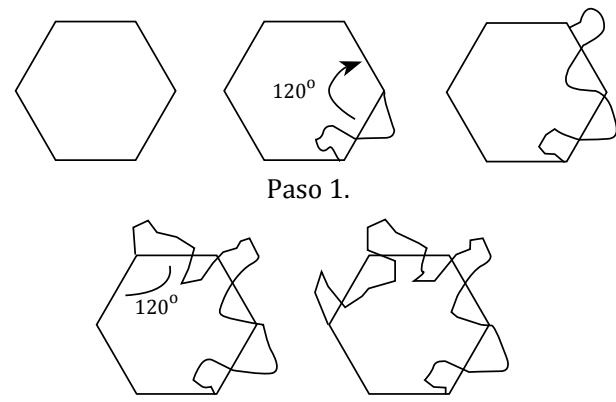

Figura 14. Los dos primeros pasos para construir el lagarto de la baldosa básica

Este truco se puede aplicar cuando se tienen lados consecutivos iguales en el polígono de la teselación original ${ }^{7}$.

\section{Los grupos p2, p4, p6 y los calidoscopios S2222, S244 y $\mathbf{S}_{236}$}

El calidoscopio S2222, como su notación lo indica, tiene cuatro esquinas que son conos de $180^{\circ} \mathrm{y}$ aparece en dos formas básicas diferentes una es la de una almohada tradicional y la otra tiene forma de cojín tetraédrico o almohada torcida. Este calidoscopio corresponde al grupo cristalográfico p2, que es generado por cuatro rotaciones de $180^{\circ}$. Representamos la construcción de la almohada torcida junto con su diseño simétrico en la figura 15.

Otra forma de construir el cojín tetraédrico o almohada torcida es la siguiente: elaboramos un triángulo acutángulo sobre material flexible al cual le marcamos los puntos medios de sus tres lados. Trazamos el triángulo que une estos puntos medios, doblamos a lo largo de los lados de este nuevo triangulo y al pegar estas mitades nos produce el tetraedro. Observamos que por la forma

7 Véase el mismo truco aplicado en la construcción de otros teselados(en particular, los teselados 104 y 99) en http://britton.disted.camosun.bc.ca/jbescher4. htm 
en que lo hemos construido, cada vértice de este tetraedro está formado por tres ángulos cuya suma es exactamente $180^{\circ}$. Le proponemos al lector construir este tetraedro con papel. Si dicho tetraedro se cubre con tinta en forma irregular y se echa a rodar sobre un plano se producirá un diseño simétrico.

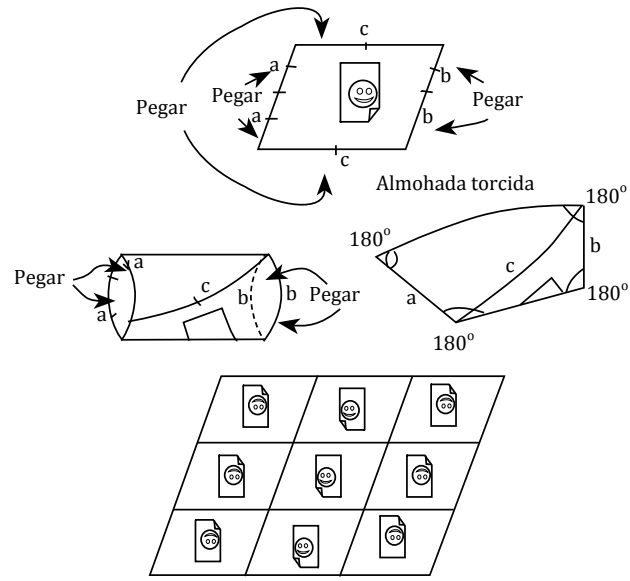

Figura 15.S2222 en forma de almohada torcida

Por su lado, los calidoscopios $\mathrm{S}_{244} \mathrm{y}$ S236 (figura 16) son análogos al cojín S333 y tienen forma de cojines triangulares. Ellos corresponden a los grupos cristalográficos p4 y p6. Donde p4 es generado por tres rotaciones, una de $180^{\circ} \mathrm{y}$ dos de $90^{\circ} \mathrm{y}$, por su lado, p6 también es generado por tres rotaciones, en este caso de $180^{\circ}, 120^{\circ}$ y $60^{\circ}$.
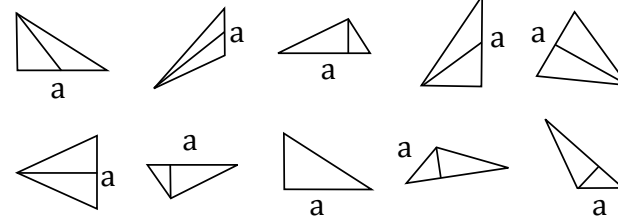

Figura 16. Los cojines S244 y S236

En la obra de Escher encontramos diseños con estos tres calidoscopios. Por ejemplo, Ardillas $^{8}$ y Sapos $^{9}$ (Frogs) corresponden ambas al calidoscopio S2222.

Por otro lado, la obra titulada Pescado, de 1938, y la del mismo nombre, de 1964 , son ambas producidas por $\mathrm{S}_{244}{ }^{10}$. De la misma forma, la obra Lagartos II (teselado 104) corresponde a $\mathrm{S}_{244}{ }^{11}$. En la página web http://britton.disted. camosun.bc.ca/jbescher4.htm, tenemos un ejemplo de $\mathrm{S}_{236}$ en la obra del Pez volador (haga clic en el teselado 99 para ver el diseño completo). Es interesante observar allí mismo la animación de la construcción de su baldosa básica.

\section{Los grupos $\mathrm{cmm}$, pmg y los calidoscopios $D_{\overline{222}}$ y $D_{22}$}

A continuación describiremos cómo se construyen los calidoscopios $D_{\overline{222}}$ y $D_{22}$. Primero, consideremos la almohada rectangular S2222, sin diseño pintado sobre ella. Pensémosla como si estuviese llena de aire. Ella tiene un plano de simetría que pasa simultáneamente por las cuatro esquinas. Al cortar la almohada a lo largo de este plano, obtenemos dos piezas rectangulares del mismo tamaño. Si pensamos que ellas son hechas de material poroso, entonces cada una de ellas resulta ser el calidoscopio $D_{\overline{2222}}$ estudiado anteriormente. Así, de $\mathrm{S}_{2222}$ hemos obtenido dos veces $\mathrm{D}_{\overline{2222}}$, por esta razón se dice que S2222 $_{22}$ es el doble de $D_{\overline{2222}}$. Pero $S_{2222}$ admite otras dos

\footnotetext{
8 Véase http://www.tessellations.org/eschergallery2. htm

9 Véase http://www.tessellations.org/eschergallery14.htm

10 Ver http://www.tessellations.org/eschergallery 10. htm y http://www.tessellations.org/eschergallery26.htm

11 En http://britton.disted.camosun.bc.ca/jbescher4. htm se aprecia la animación de la baldosa básica de Lagartos II
} 
clases de planos de simetría (figura 17) y cada vez que cortemos por uno de ellos encontraremos otro artefacto que si lo pensamos de material poroso nos permitirá imprimir diseños diferentes.

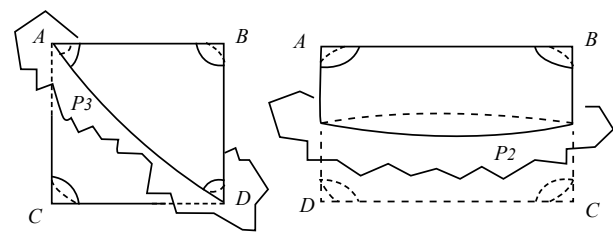

Figura 17. Otros planos de simetría deS 2222

$\mathrm{Al}$ cortar por los planos anteriores obtenemos los calidoscopios $D_{222}$ y $D_{22}$, donde el primero de ellos se parece a un sombrero de fiesta de cumpleaños con dos ángulos de $90^{\circ}$ en su borde y el segundo a una funda de almohada. Apreciamos estos calidoscopios en la figura 18, junto con los diseños simétricos que ellos producen. De la misma forma, los números de la notación nos indican la esencia de los calidoscopios. Así, el número 2 sin barra de $D_{2 \overline{22}}$ nos dice que hay un centro de rotación de $180^{\circ}$; la letra $\mathrm{D}$, que hay reflexiones, y los dos números 2 con barra, que hay dos vértices diferentes donde se tienen reflexiones a lo largo de ejes perpendiculares entre sí, que son justamente ángulos de $90^{\circ}$ en el borde del disco. Por otro lado, en la notación $D_{22}$, la letra D indica la existencia de un eje de reflexión y los dos números 2 , indican la existencia de dos centros de rotación de $180^{\circ}$. La obra Cangrejos ${ }^{12}$ (Crabs) es un ejemplo de $\mathrm{D}_{22 .}$.
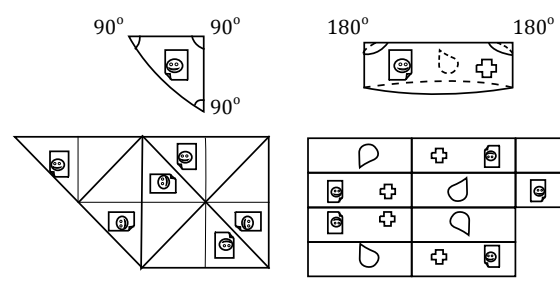
Figura 18. Los calidoscopios $D_{\overline{222}}$ y $D_{22}$ y sus
diseños simétricos

\section{Los grupos p4g, p31 m y los calidoscopios $D_{4 \overline{2}}$ y $D_{\mathbf{3}}$}

Los artefactos $D_{4 \overline{2}}$ y $D_{3 \overline{3}}$ correspondientes a los grupos p4g y p31m se asemejan también a sombreros de fiesta de cumpleaños que aparecen al cortar, respectivamente, los cojines triangulares $\mathrm{S}_{244}$ y $S_{333}$ a lo largo de planos de simetría (figura 19).
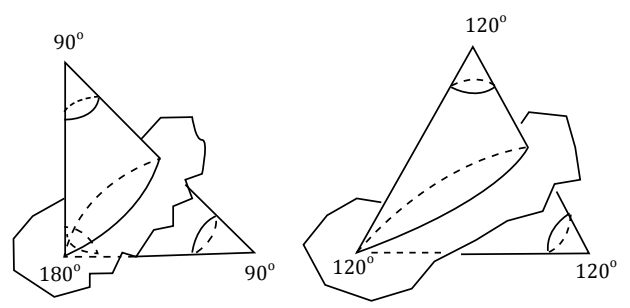

Figura 19. Planos de simetría de $\mathrm{S}_{244}$ y $\mathrm{S}_{333}$

De nuevo $D_{4 \overline{2}}$ indica que se tiene un cono de $90^{\circ}$ y que hay un ángulo de $90^{\circ}$ en el borde. Mientras que $D_{3 \overline{3}}$ tiene un cono de $120^{\circ}$ y un ángulo de $60^{\circ}$ en el borde. De esto podemos deducir que p4g se puede generar con una rotación de $90^{\circ}$ junto con dos reflexiones a lo largo de ejes perpendiculares y, por su parte, p31m se genera por una rotación de $120^{\circ}$ junto con dos reflexiones a lo largo de ejes que forman un ángulo de $60^{\circ}$ (figura 20).

12 Véase en http://www.mcescher.com/indexuk.htm, página oficial de Escher 


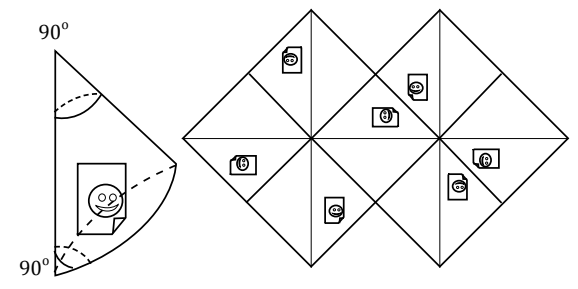

$60^{\circ}$

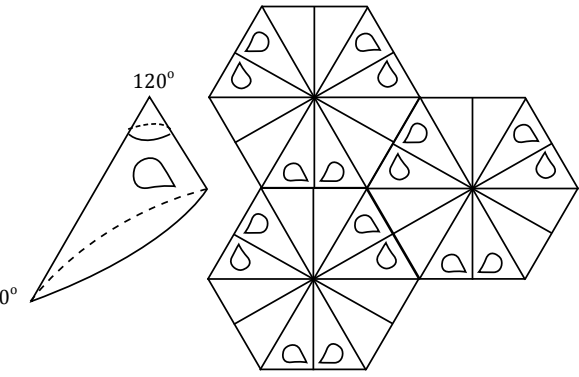

Figura 20. $D_{4 \overline{2}}$ y $D_{3 \overline{3}}$ con sus diseños simétricos

En Escher encontramos diseños con estos calidoscopios. Por ejemplo, las obras Hombres fuertes ${ }^{13}$ y Ángeles y demonios ${ }^{14}$ corresponden al calidoscopio $D_{4 \overline{2}}$. Por otro lado, El niño chino ${ }^{15}$, de 1936, se relaciona con $D_{3} \overline{\text {. }}$.

\section{Los grupos pg, pgg y los calidoscopios K y P22}

Llegamos al final del listado de calidoscopios y, con ello, a los diseños de Escher más complejos y algunos de los más bellos. Los calidoscopios $\mathrm{K}$ y $\mathrm{P}_{22}$ son llamados, respectivamente, botella de Klein y plano proyectivo. K primera letra de Klein y P primera letra de proyectivo.

Las obras Golondrinas (Swallows) y Jinetes $^{16}$ (Horsemen), así como Leones alados (Wingedlions, en el teselado 66) y
Bulldogs ${ }^{17}$ (en el teselado 97) son ejemplos de la botella de Klein K.

Al observarlas cuidadosamente se detecta en ellas dos reflexiones corridas sobre ejes paralelos. Estas reflexiones se ven muy claras en la animación (que se aprecia en la misma página web) de la construcción de la baldosa básica de Leones alados. Es interesante anotar que en la construcción de la baldosa de Bulldog no se observa sino una única reflexión corrida la cual es seguida por una traslación. Sin embargo, así como el grupo pg, correspondiente a $\mathrm{K}$, se puede generar por dos reflexiones corridas paralelas, también es posible generarlo con una sola reflexión corrida y una traslación.

Teniendo en cuenta la última observación, el artefacto $\mathrm{K}$ debe ser la combinación de un cilindro, que produce traslaciones, con una cinta de Möebius que produce una reflexión corrida. Para construirlo partimos de un rectángulo con cierres en sus bordes, tal cual lo ilustramos en la figura 21.

El proceso de impresión consiste en cerrar primero los cierres marcados con la letra A, hacer rodar la cinta de Möebius, para imprimir una cinta horizontal, y luego abrir los cierres A y cerrar los $\mathrm{B}$, para hacer rodar una vuelta completa el cilindro; luego se intercambian los cierres, para imprimir de nuevo con la cinta de Möebius, y así se continúa imprimiendo alternando los cierres.

\footnotetext{
13 Véasehttp://www.tessellations.org/eschergallery3.htm

14 Véase http://www.dartmouth.edu/ matc/math5. pattern/lesson7art.html

15 Véasehttp://www.tessellations.org/eschergallery1.htm

16 Ambas obras se aprecian en http://www.tessellations.org/eschergallery2thumbs.htm
}
17 Se aprecian en http://britton.disted.camosun.bc.ca/ jbescher4.htm




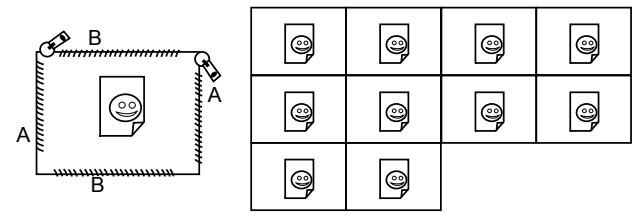

Figura 21. Botella de Klein y su diseño

Para terminar esta sección de teselados planos, estudiamos el plano proyectivo $\mathrm{P}_{22}$, que es el calidoscopio correspondiente a las obras Leones (Lions) y Perros (Dogs) ${ }^{18}$. Al detallar estas obras, encontramos que en cada una de ellas hay dos reflexiones corridas con respecto a ejes perpendiculares. Su grupo cristalográfico correspondiente es pgg. El artefacto que imprimirá un diseño de estos debe ser una combinación de dos cintas de Möebius, que deben trabajar en forma perpendicular. Al igual que en el caso anterior, partimos de un rectángulo con cierres en sus bordes dispuestos como en la figura 22. Y la operación del artefacto se hace alternando el uso de los cierres, unas veces para obtener una cinta de Möebius que imprime en forma vertical y otras veces una cinta de Möebius que imprime en forma horizontal.

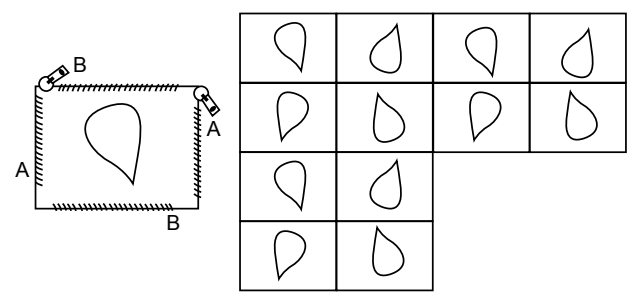

Figura 22. Plano proyectivo y su diseño

18 Véase http://www.tessellations.org/eschergallery1 thumbs.htm

\section{Teselados en otras curvaturas}

Así como tenemos teselados sobre un plano, es posible considerar los sobre otras superficies. Un plano es una superficie de curvatura constante cero. Una esfera es una superficie de curvatura constante positiva y un plano hiperbólico es una superficie de curvatura constante negativa. La figura 23 nos muestra teselados hechos con triángulos en cada una de estas curvaturas.
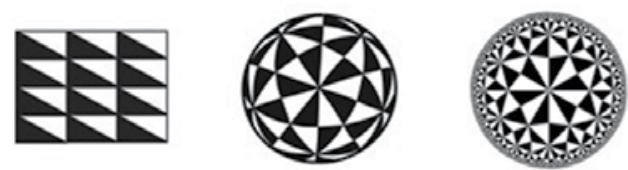

Figura 23. Teselados sobre superficies de distintas curvaturas

Cuando observamos las dos primeras figuras, nuestra mente reconoce que la primera de ellas cubre una superficie plana (euclídea) y que la segunda cubre una esfera. Con respecto a la tercera nuestro cerebro no tiene la experiencia y se confunde. Manteniendo la mirada sobre ella vemos a veces que los vértices de los triángulos se salen del plano y otras que se hunden en el plano, y no se sabe exactamente de qué superficie se trata; esta última es un teselado del modelo de Poincaré del plano hiperbólico.

\section{Teselados esféricos}

La mayoría de los teselados que hizo Escher son euclídeos, pero también podemos encontrar en sus trabajos teselados sobre esferas. Son ejemplos una esfera esculpida en madera y teselada con peces ${ }^{19}$, al igual que otra bella esfera (también esculpida en madera) con úntese lado hecho de ángeles y demonios,

19 Véase http://www.dartmouth.edu/ matc/math5. pattern/lesson7art.html 
cuya fotografía se encuentra en Ernst (1978, p. 41). También ubicamos un falso teselado de una esfera ${ }^{20}$; decimos que es falso porque la figura del pez cambia de tamaño sobre la esfera, lo cual no es permitido en un verdadero teselado, ya que los movimientos son rígidos. Los teselados esféricos pueden también ser producidos por artefactos similares a los calidoscopios ya discutidos.

\section{Teselados hiperbólicos}

Escher estuvo muy obsesionado con la posibilidad de plasmar el infinito en una superficie finita; idea que lo llevó a hacer muchos teselados del plano hiperbólico usando principalmente el modelo de Poincaré. El disco de Poincaré es una especie de claraboya redonda que nos permite visualizar el plano hiperbólico. Tiene la particularidad que a medida que nuestros ojos se dirigen al borde de la claraboya las figuras que observamos parecen cada vez más lejos; por eso, su tamaño aparente es más pequeño. El borde corresponde al infinito.

En el teselado del plano hiperbólico (tercera figura) de la figura 23 debemos entender que todos los triángulos tienen el mismo tamaño, solo que los que vemos más pequeños están más alejados. En cada una de las obras Límite circular III, Límite circular IV ${ }^{21}$ y Límite circular $\mathrm{I}^{22}$ debemos entender que las figuras son del mismo tamaño aunque parezcan de diferentes dimensiones.

\footnotetext{
20 Véase http://aixa.ugr.es/escher/800x600/Superficie_esferica_con_peces.jpg

21 Ésta y la anterior se pueden apreciar en http://www. arrakis.es/ sysifus/escher.html

22 Véase http://aixa.ugr.es/escher/800×600/Limite_circular_l.jpg
}

Para visualizar el plano hiperbólico existen diferentes clases de ventanas (o modelos). Además del modelo del disco de Poincaré, Escher usó el modelo del semiplano superior en la obra División de superficie ${ }^{23}$. Lo mismo ocurre en la obra División ${ }^{24}$. En este modelo el borde inferior está en el infinito y, cuanto más cerca estén las figuras a dicho borde ellas realmente están más lejos; por esa razón su tamaño aparente es más pequeño.

Antes de enterarse de la existencia del modelo de Poincaré, Escher venía trabajando en su obsesión de plasmar el infinito en sus dibujos. En trabajos como Evolución II $^{25}$ y Pequeño y más pequeño ${ }^{26}$, Escher estaba elaborando la misma idea del infinito pero organizó las cosas de tal manera que las figuras más distantes estaban más cerca al centro del dibujo, por consiguiente, su tamaño es cada vez más pequeño cuanto más cerca estén del centro. Estos dibujos los podemos pensar como la visión que tendríamos a la entrada de un túnel que está teselado. En su última y magistral obra Serpientes ${ }^{27}$ combina lo que ocurre en el disco de Poincaré con la idea de túnel teselado. Allí vemos que, aproximándonos tanto al borde como al centro del círculo mayor, los círculos que tejen la maya donde se enredan las serpientes se achican, dando la impresión de lejanía.

\footnotetext{
23 Se aprecia en http://aixa.ugr.es/escher/800x600/ Division_de_la_superficie.jpg

24 Véase http://www.tessellations.org/eschergallery21.htm

25 Véase http://aixa.ugr.es/escher/800×600/Evolucion_ll.jpg

26 Véase http://www.tessellations.org/eschergallery22.htm

27 Véase http://aixa.ugr.es/escher/800x600/Serpientes.jpg
} 
(Para más información sobre el plano hiperbólico y el modelo de Poincaré, consúltesehttp://mathworld.wolfram.com/ PoincareHyperbolicDisk.html yhttp:// math.youngzones.org/Non-Egeometry/ poincare.html. Véase también Montesinos-Amilibia, 1987, 1992 y RamírezGalarza y Seade, 2002).

\section{Teselados del espacio}

Así como un plano euclídeo se puede teselar, es posible teselar el espacio euclídeo. En este caso las baldosas básicas que se repiten son tridimensionales. Aunque brevemente, Escher también incursionó en el campo de teselados del espacio euclídeo tridimensional. Sus obras División cúbica del espacio ${ }^{28}$ y Profundidad ${ }^{29}$ hacen gala de su impresionante capacidad geométrica y son ejemplo de teselados del espacio euclídeo.

\section{Teselados como partes de otras obras}

Muchas de los teselados que realizó Escher eran estudios preliminares para introducirlas como parte de otras obras mucho más complejas.

En la obra Reptiles ${ }^{30}$ vemos que se incluye, como parte de ella, el teselado plano Reptiles (boceto) mencionada en el estudio de $S_{333}$. Esta obra plasma cómo Escher se maravillaba con la idea de poder hacer dibujos con la apariencia de tres dimensiones en una superficie de solo dos dimensiones. De forma similar y magistralmente, Escher usa el teselado

28 Véase http://aixa.ugr.es/escher/800×600/Division_cubica_del_espacio.jpg

29 Véase en http://www.mcescher.com/indexuk.htm, página oficial de Escher

30 Véase http://britton.disted.camosun.bc.ca/jbescher2.htm de los Leones alados como parte central de su obra El espejo mágico ${ }^{31}$, donde de nuevo se puede apreciar el juego de las tres dimensiones en dos dimensiones. Esta idea se repite en Encuentro ${ }^{32}$, siendo el teselado del fondo también una Botella de Klein ${ }^{33}$.

La serie de Metamorfosis (I, II y III) además de utilizar algunos teselados ya estudiados por él, involucra el desarrollo del concepto topológico de homotopía. Informalmente podemos decir que una homotopía es la de formación continua de un objeto durante un determinado periodo.

En Metamorfosis $\mathrm{I}^{34}$, Escher retoma el teselado del Niño chino (véase el estudio de $D_{3 \overline{3}}$ y la transforma gradualmente en un pueblo al lado de un acantilado. En Metamorfosis $\mathrm{II}^{35}$ se observa la transformación de la palabra metamorphose pasando por diversos teselados y dibujos.

De carácter muy similar es la obra Metamorfosis III, la cual se aprecia en la página web: http://en.wikipedia.org/ wiki/Metamorphosis_III

\section{Conclusiones}

Hemos visto en este artículo varias cosas:

- Los grupos cristalográficos desde un punto de vista geométrico y práctico.

31 Véase http://aixa.ugr.es/escher/800x600/Espejo_magico.jpg

32 (http://www.tessellations.org/eschergallery17.htm)

33 Véase http://www.mcescher.com/indexuk.htm, la galería de pinturas de la página oficial de Escher

34 Véase http://www.tessellations.org/eschergallery6. $\mathrm{htm}$

35 Véase la animación que recorre esta obra en http:// www.3quarks.com/Applets/Metamorphose/ 
- Una forma divertida para entender los movimientos rígidos.

- Una forma práctica para imprimir diseños simétricos.

- Una introducción al concepto de orbifold.

- La obra de Escher como ejemplificación de los grupos cristalográficos.

\section{Referencias}

Albis-González, V. y Valencia-Oviedo, J. (1990). Una aplicación de los grupos de simetría a la confirmación de periodos y sub periodos estilísticos en la cerámica de la región central de Panamá. Revista de la Academia Colombiana de Ciencias, 17(67), 703-714.

Ernst, B. (1978). Le Miroirmagique de M.C. Escher. Berlín: Ed. Benedikt Taschen.

Ernst, B. (1991). Un mundo de figuras imposibles. Berlín: Ed. Benedikt Taschen.

Gutiérrez-Santos, M. (1992). Notas de geometría. Bogotá: Universidad Nacional de Colombia.

Hilden, H.; Montesinos, J.; Tejada, D.M. y Toro, M.M. (2011). Artifacts for stamping symmetric designs. American Mathematical Monthly, 118(4), 327-343.

Klein, C. y Hurlbut, C. (1999). Manual of mineralogy. Nueva York: John Wiley and Sons Inc.

Montesinos- Amilibia, J. (1987a). Classical tessellations and three-manifolds. Berlín-Heidelberg: Springer-Verlag.
- Una introducción rápida a otras curvaturas.

- Interpretaciones diferentes, a las usuales en topología, del toro, de la cinta de Möebius, de la botella de Klein y del plano proyectivo.

Montesinos-Amilibia, J. (1987b). Caleidoscopios y grupos cristalográficos en la Alhambra. Revista Epsilón. 9, 9-30. Montesinos-Amilibia, J.M. (1992). Las geometrías no euclídeas. En: G. Lobachevski y Bolyai. Historia de la Matemática en el siglo XIX (pp. 65-114). Madrid: Real de Academia de Ciencias.

Montesinos-Amilibia, J. (2003). Calidoscopios y 3-variedades. Bogotá: Universidad Nacional de Colombia.

Montesinos-Amilibia, J. (4 de agosto de 2005). Geometría en los Mosaicos del Palacio de la Alhambra de Granada. Conferencia, Cátedra Pedro Nel Gómez. Video Institucional. Bogotá: Universidad Nacional de Colombia.

Ramírez-Galarza, A. y Seade, J. (2002). Introduction to classical geometries. Berlín: Birkhauser Verlag.

Seymour, D. y Britton, J. (1989). Introduction to tessellations. Palo Alto, California: Dale Seymour Publications.

Thurston, W. (1997).Three-dimensional geometry and topology. Princeton, $\mathrm{NJ}$ : Princeton University Press. 